\title{
Varicocele treatment: A 2-centers comparison between non microsurgical open correction, laparoscopic approach and retrograde percutaneous sclerotization on 463 cases
}

\author{
Giangiacomo Ollandini ${ }^{1}$, Giovanni Liguori ${ }^{1}$, Stanislav Ziaran ${ }^{2}$, Tomáš Málek ${ }^{2}$, \\ Giorgio Mazzon ${ }^{1}$, Bernardino de Concilio ${ }^{1}$, Stefano Bucci ${ }^{1}$, Sara Benvenuto ${ }^{1}$, \\ Emanuele Belgrano ${ }^{1}$, Carlo Trombetta ${ }^{1}$ \\ ${ }^{1}$ Urologic Unit, Cattinara Hospital, University of Trieste, Trieste, Italy; \\ ${ }^{2}$ Urology clinic, University hospital in Bratislava, Comenius University, Bratislava, Slovakia.
}

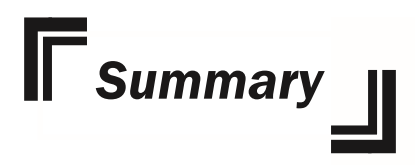

Objectives: To determine whether there are differences in sperm parameters improvement after different varicocele correction techniques. To determine the role of age in sperm parameters improvement.

Methods: 2 different European centers collected pre- and postoperative sperm parameters of patients undergoing varicocele correction. Among 463 evaluated patients, 367 were included. Patients were divided in procedure-related and age-related groups. Ivanissevich inguinal open surgical procedure (OS), lymphatic-sparing laparoscopic approach (LSL) and retrograde percutaneous transfemoral sclerotization (RPS) were performed. As outcome measurements sperm count (millions/mL, SC) and percentage of mobile sperms were analyzed. Univariate and multivariate regression between the defined groups; bivariate regression analysis between age and sperm count and motility.

Results: Number of patients: OS 78; LSL 85; RPS 204. Mean age 30.2 (SD 6.83); postoperative SC increased from 18.2 to 30.1 (CI 95\% 27.3-32.9; $p<0,001$ ); motility from 25.6 to $32.56 \%$ (30.9-34.2; $p<0.001)$. OS: SC varied from 16.9 to 18.2 ( $p<0.001)$; sperm motility from $29 \%$ to $33 \%$ ( $p<0.001)$. LSL: SC from 15.5 to 17.2 ( $p<0.001)$; motility from 27 to $31 \%(p<0.001)$. RPS: SC from 18.9 to 36.2 ( $p<0.001)$; motility from $24 \%$ to $32 \%(p<0.001)$. Univariate and multivariate analysis confirmed the significant difference of SC variation in RPS, compared to the other groups $(p<0.001)$. No significance between LSL and OS $(p=0.826)$. No significant differences regarding motility $(p=0.8)$.

Conclusions: Varicocele correction is confirmed useful in improving sperm parameters; sclerotization technique leads to a better sperm improvement compared to other studied procedures; improvement in seminal parameters is not affected by age of the patients treated.

KEY WORDS: Andrology; Infertility; Sclerotization; Spermatozoa; Varicocele.

\section{INTRODUCTION}

Varicocele can affect all parameters of sperm characteristics, including sperm count, sperm motility, and morphology. Different techniques have been suggested for varicocele treatment, both surgical and non-surgical. The surgical techniques include the open surgical (inguinal, subinguinal, retroperitoneal approach), laparoscopic and microsurgical varicocelectomies. The non-surgical are represented by the radiological-assisted techniques: embolization or sclerotherapy $(1,2)$.

Several studies related the outcome in terms of invasiveness and costs between the different techniques used $(3,4)$; however a comparison of the efficacy on sperm parameters improvement of open ligation, laparoscopic approach and sclerotization is still missing. 
The goal of our study was to compare the clinical outcomes in terms of sperm quality after varicocele correction using the three cited techniques. We also referred to patients' age in order to analyze whether there are significant differences of postoperative sperm improvement related to age increment.

\section{Material AND METHODS}

Two different centers collected data of patients undergoing varicocele treatment from 1986 to 2011. Patients were complaining both from infertility or testicular pain. All patients underwent a complete history, physical examination in a warm room, hormonal assessment, semen analysis. Each center treated the patients with a different technique: open ligation of the spermatic vein according to Ivanissevich technique, laparoscopic approach or retrograde sclerotization of the spermatic vein. At least 3 months after surgery, semen analysis and physical examination have been performed: in fact $\mathrm{Al}$ Bakri et al. in 2012 demonstrated that there is no significant improvement in sperm parameters after 3 months from correction (6).

On a total of 463 patients, 96 have been excluded according to the following criteria: persistence of varicocele, endocrinological abnormalities, history of undescended testis, bilateral varicocele, and abnormal right testis. Mean age of patients was 30.2 yr (SD 6.83); median clinical grade was 2 (IQR 1); mean sperm concentration was 18.0 millions/mL (SD 14.7) and mean sperm motility was $25.6 \%$ (SD 17.51).

Surgical procedures: the operative procedures are widely described in the literature.

In Ivanissevich open surgery inguinal approach (under general anesthesia) the exposure of the internal spermatic vessels within the inguinal canal takes place through an incision of the external oblique aponeurosis (7).

In laparoscopic ligation of spermatic veins (under general anesthesia) the patients underwent varicocelectomy by the lymphatic sparing technique. In this procedure the internal spermatic veins alone were divided. Using a microsurgical technique both the artery and the lymphatics were preserved (8).

Patients underwent retrograde percutaneous sclerotization of their left spermatic vein, using the right transfemoral retrograde percutaneous approach. The femoral vein is entered below the inguinal ligament using the standard Seldinger technique. Renal phlebography is carried out by injection of contrast medium under Valsalva maneuver. After superselective catheterization of the spermatic vein, a guidewire is introduced and act as a guide for a very distal catheterization, through continuous fluoroscopy. Superselective angiography shows every possible collateral circle and the possible presence of multiple spermatic veins. Sclerotization technique is performed by injecting a 2-4 $\mathrm{mL}$ of sodium tetradecyl sulfate 3\% mousse. Patients are required to perform a Valsalva maneuver at least 10 seconds long during the injection. Venography is then performed again: should there be bulky veins, the operation is repeated at a higher lumbar level. After this procedure, a control venography is performed to confirm the absence of renosper- matic reflow $(9,10)$. It is known from the literature that up to $20 \%$ of patients have anatomical abnormalities of their veins, that could eventually make not possible the retrograde technique. When this happened, we performed anterograde sclerotization according to Tauber technique during the same session (11).

\section{Sermadysis}

Specimens were obtained by masturbation after 3 to 5 days of abstinence. The specimens were valuated within 1 hour from collection for the following parameters: sperm concentration (millions $/ \mathrm{mL}$ ), percentage of sperms with A + B motility (A, speed linear motility; B, slow linear motility; C, motility in situ; D, no motility at all), percentage of morphologically typical sperms. The laboratories evaluated the parameters according to WHO criteria.

\section{Staisticd adysis}

Statistical analyses were performed with SPSS 17.0 software package. Description of population and parameters have been reported as mean values with standard deviation (SD) for continuous variables, and with median values with interquartile range (IQR) for non-continuous ones. The significances of differences between preoperative and postoperative values within groups have been valued with the paired Student t-test, if appropriate, or with the Wilcoxon signed rank test. Means variations between two groups has been valuated with MannWhitney $\mathrm{U}$ test. ANOVA univariate analysis of variance with LSD post-hoc evaluation has been carried out in order to compare mean values of more than two groups. MANOVA Multivariate analysis has been then performed between our data.

The linear regression coefficients have been calculated in order to test the correlation between age and parameters. Probability values $<.05$ were considered significant.

\section{RESULTS}

Patients included in the study were a total of 367. Among these, 78 underwent open surgical approach; 85 were treated by laparoscopy and 204 by sclerotization technique. Persistence and minor complication rates are shown in Table 1. No major complications occurred. Complications in surgical ligation of spermatic vein were hydrocele, difficult wound healing and hematomas. Complications among sclerotization techniques were mostly represented by persistent (more than 3 days) pain at the spermatic chord. Due to the injection of sclerosing substance, though, self-recovery pain and an acceptable increase of volume and consistence of the chord was not considered as a complication.

Sperm concentration increased postoperatively in $73 \%$ of global cases; motility from 25.6 to $32.56 \%$ $(\mathrm{p}<0.001)$. Average postoperative sperm concentration increased to 30.1 millions/mL (SD 29.9; CI 95\% 27.3$32.9 ; \mathrm{p}<0.001$ ) and motility to $32.56 \%$ (SD 17.3; CI 95\% 30.9-34.2; $\mathrm{p}<0.001$ ).

Patients have been divided into three groups, according to the procedure performed, and into 4 groups, according to their age. Every difference within the groups obtained a significance p-value $<0.001$. 
Table 1.

Total number of treated and excluded and rate of overall post-operative complications.

\begin{tabular}{|c|c|c|c|c|c|c|}
\hline Technique & $\mathrm{N}$ & & Excluded & & Included & Complications \\
\hline & & Other reasons & Recurrency (\%) & Total & & \\
\hline Open Surgery & 77 & 2 & $7(9,1 \%)$ & 9 & 68 & $7 \%$ \\
\hline Laparoscopic & 80 & 4 & $1(1,3 \%)$ & 5 & 75 & $0 \%$ \\
\hline Sclerotization & 366 & 57 & $25(9,3 \%)$ & 82 & 284 & $5 \%$ \\
\hline Total & 523 & 63 & 33 & 96 & 427 & \\
\hline
\end{tabular}

Table 2.

Univariate ANOVA and Multivariate MANOVA analysis of variance.

\begin{tabular}{|c|c|c|c|c|c|c|}
\hline & & \multirow[t]{2}{*}{ Age (SD) } & \multicolumn{2}{|c|}{ Sperm Concentration (95\% Cl) } & \multicolumn{2}{|c|}{ Motility (95\% Cl) } \\
\hline & & & Pre & Post* & Pre & Post* \\
\hline \multirow[t]{3}{*}{ Procedure } & Open Surgery & $32,1(6,85)$ & $16,9(13,4-20)$ & $18,2(15,0-21,5)$ & $29(25,8-32)$ & $33,1(30,9-35,4)$ \\
\hline & Laparoscopy & $26(5,6)$ & $15,5(13,7-17,1)$ & $17,2(15,5-18,9)$ & $27(23,5-29,6)$ & $31,2(28,7-33,8)$ \\
\hline & Sclerotization & $25,9(5,2)$ & $18,9(17-20,1)$ & $36,2(32,8-40,7)$ & $24(21,3-29,9)$ & $32,9(30,3-35,6)$ \\
\hline \multirow[t]{2}{*}{ p-value } & Univariate & & 0,2 & $<0,0001$ & 0,25 & 0,21 \\
\hline & Multivariate & & 0.18 & $<0,0001$ & 0.18 & 0.15 \\
\hline \multirow[t]{4}{*}{ Age decades } & Group 1 & $0-20$ & $18,8(13,7-23,9)$ & $27,3(25,7-38,8)$ & $30,6(26,1-35,2)$ & $38,5(33,9-43,0)$ \\
\hline & Group 2 & $21-30$ & $17,8(15,7-19,9)$ & $29,5(25,4-33,6)$ & $26,3(24,0-28,5)$ & $33,8(31,5-36,2)$ \\
\hline & Group 3 & $31-40$ & $17,2(15-19,4)$ & $30,9(26,2-35,5)$ & $24,1(21,2-27,0)$ & $30,9(28,1-33,7)$ \\
\hline & Group 4 & $>41$ & $22,5(17,1-27,9)$ & $36,4(28,2-44,6)$ & $23,2(16,0-30,3)$ & $30,2(24,7-35,7)$ \\
\hline \multirow[t]{2}{*}{$\overline{p \text {-value }}$} & Univariate & & 0,85 & 0,32 & 0,08 & 0,004 \\
\hline & Multivariate & & 0.97 & 0.30 & 0.13 & 0.003 \\
\hline
\end{tabular}

${ }^{*} p$-value $<0,001$ for each of the parameters withing the groups.

Figure 1.

Mean variation of sperm concentration and motility between the three procedure-related groups.

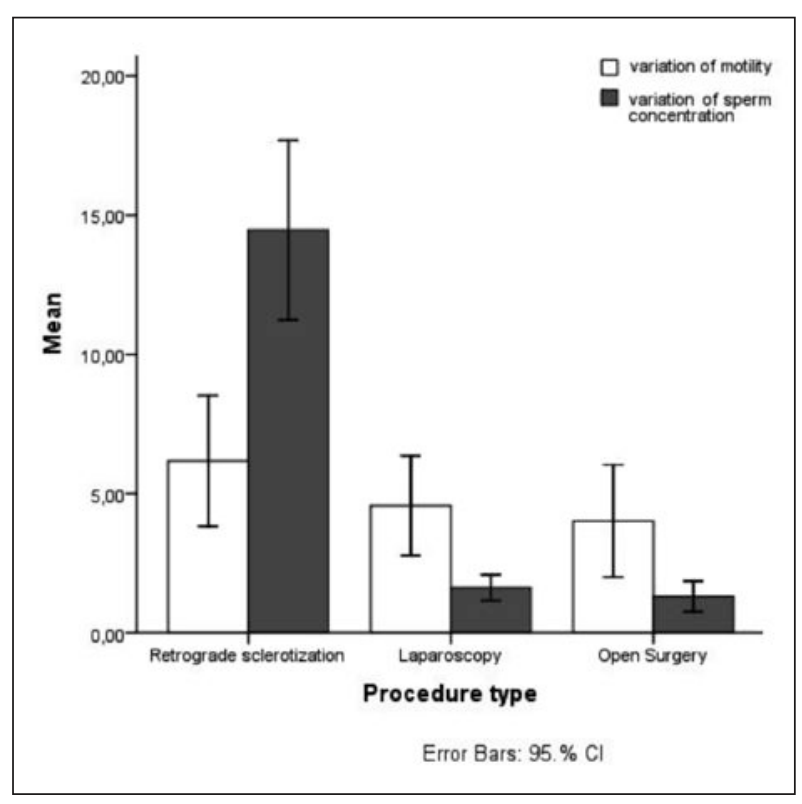

\section{Iifferences latweenthe gaus}

Univariate ANOVA regression analysis and multivariate MANOVA have been performed in order to evaluate the differences between the groups and set their significance (Table 2). Post hoc evaluation of variances between procedure-related groups have been reported for sperm count. Variation of sperm count has been demonstrated to be related to the procedure (Figure 1), being significantly higher for patients treated by sclerotization procedure.

Age decades showed no significant differences in mean values of sperm count and sperm motility improvement (Table 2). Mean variation of sperm concentration showed also no significant differences between age-related groups (Figure 2).

The linear regression standardized coefficient between age and sperm motility for preoperative values is -0.09 $(p=0.048)$ and for postoperative values is -0.10 $(p=0.021)$. Therefore there is no significant difference between the calculated coefficients (Figure 3).

\section{Discussion}

In our study we found:

- As already known from the literature, varicocele correction is confirmed as useful in improving sperm parameters 
Figure 2.

Variation of sperm concentration is not significantly different among patients from different age decades.

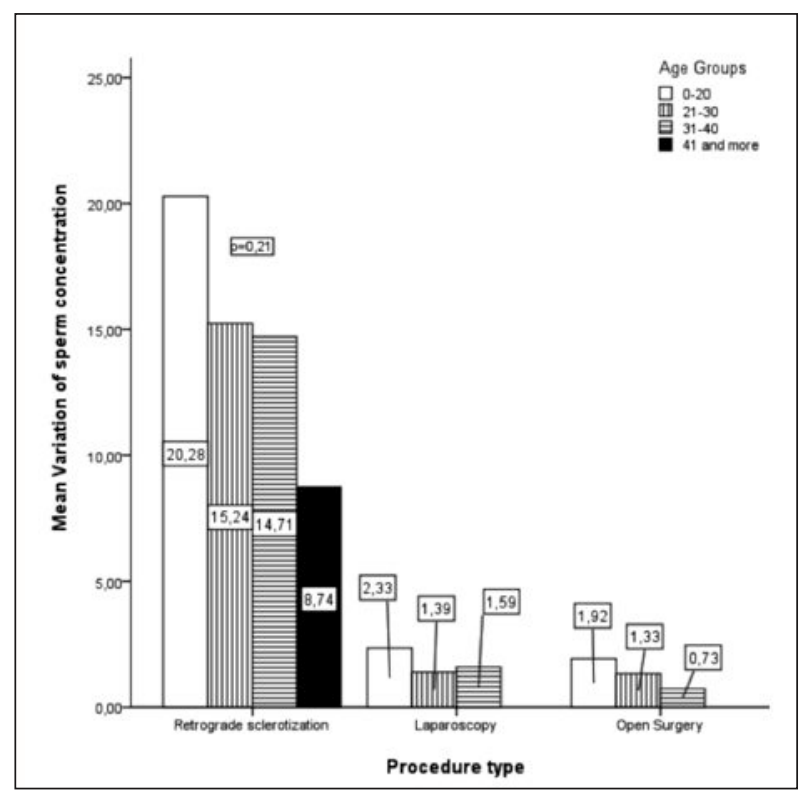

Figure 3.

Pre and postoperative percentage of motility among age groups: the negative trend remains constant.

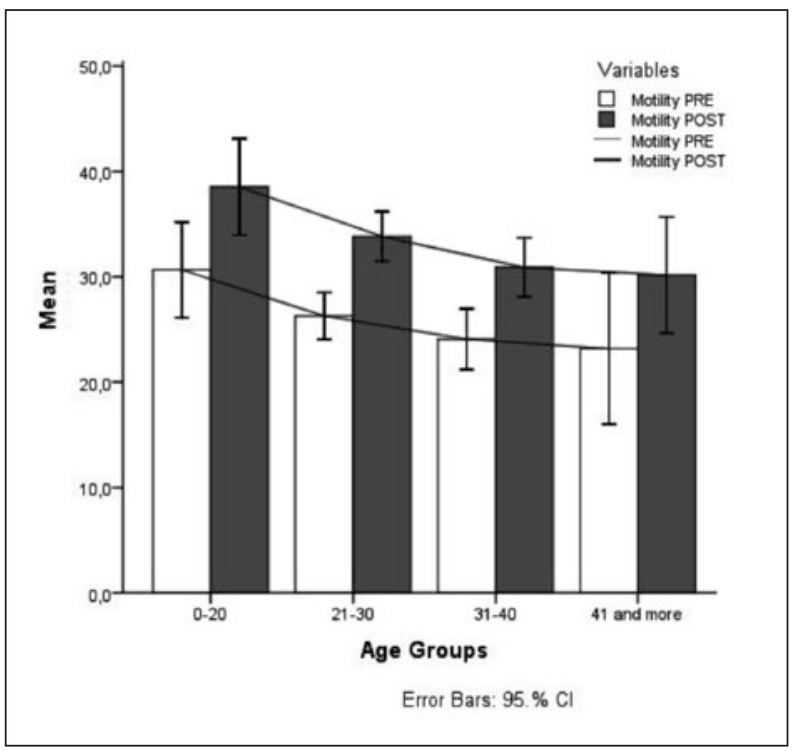

- Sclerotization technique leads to a better sperm improvement compared to other studied procedures

- Improvement in seminal parameters is not affected by age of the patients treated.

The usefulness of varicocele repair remains a highly debated topic. The 2009 updated Cochrane review by Evers and Collins discussed the indication to varicocele treatment in infertile men, and according to their metaanalysis there was no clear evidence of indication in varicocele correction to improve fertility (12).

This review, though, have been debated by a contrary opinion: the Authors (Ficarra et al.) analyzed the methodology of the study and concluded that it was weak and poorly significant, as they included patients with subclinical varicoceles and normal semen parameters (13). More recently a randomized, controlled trial by Abdel-eguid et al. (14) concluded that there is a statistically significant improvement in semen quality after microsurgical correction of varicocele and a higher pregnancy rate, comparing the results with the control arm. Therefore the main focus of our study was not to demonstrate the outcome in terms of fertility and pregnancy rate, but to compare the efficacy in sperm improvement between the different used techniques.

In 1998 Barbalias et al. carried out a randomized clinical trial comparing a total of 88 patients who underwent varicocele correction either by retroperitoneal, inguinal, subinguinal or percutaneous approach.

They analyzed pre and postoperative sperm parameters concluding that microsurgical subinguinal technique had a greater performance (15). A newer study in 2010 confirmed that microsurgical approach obtains better results compared to the standard inguinal approach (16). However microsurgical treatment of varicocele needs a greater amount of time and instruments than the other techniques. Several studies compared the open surgery technique to sclerotization procedure, with contradictory results $(12,13,17,18)$; though one of the main outcomes of those studies was the pregnancy rate, this parameter seems to be affected by too many confounding factors to be eligible as a main outcome. Many other studies, moreover, focused on the costs of the treatments, and the time to recovery: operative costs are shown to be similar for all the studied procedures, but the time to recovery is significantly less for patients treated by sclerotization technique.

Therefore Bechara et al. concluded that the radiologicalassisted procedure has a cost-benefit compared to surgical treatment (19).

The rate of technical failure of sclerotization procedure is described to vary from $5 \%$ to $20 \%$, due to the anatomical abnormalities, venospasm or technical difficulties $(13,17,19)$.

In our experience the intervention is converted during the same session to an anterograde sclerotization, according to Tauber technique. This possibility permits to obtain a $100 \%$ rate of technical success.

Our data clearly show that the sclerotization technique leads to a better improvement of sperm concentration compared to laparoscopic and Ivanissevich techniques. Moreover, these appear to obtain a similar outcome both regarding sperm density improvement and sperm motility improvement.

The hypothesis regarding the better results of sclerotization techniques are probably related to:

- Better anatomical view and complete repair of varicocele

- Complete manteinance of lymphatic vascularization

- No arterial injuries. 
Sclerotization differs from the surgical approaches because of the venography that is repeated during the whole procedure, in order to guide step by step the intervention. This allows to obtain a clear imaging of the venous vascularization, and to close selectively every single vessel that is implicated in varicocele formation. In fact it is commonly known, that 19\% of patients with varicocele have an aberrant anatomical situation (20).

This situation cannot be completely discovered by laparoscopic and surgical approach, and will be probably not treated completely, even if post operatively there is no sign of clinically detectable persistence. The pathogenetic factors involving poor sperm quality on varicocele patients, if not completely corrected, could in fact continue their damage of the testis, that have been showed in several studies $(21,22)$.

Moreover, the risk of injuries to the testicular arteries is significantly higher in patients undergoing surgical procedures than in patients undergoing sclerotization, due to the procedure itself (23), even if the role of artery injury in sperm parameter's outcome is not certainly significant $(23,24)$.

Finally, the preservation of lymphatic vessels is assured with sclerotization technique, while in patients undergoing surgical procedures the lymphatic damage is most likely avoided (laparoscopy) or most probably occurs (Ivanissevich). Lymphatic vessels ligation is thought to induce a significant worsening of testicular function, due to testicular edema (8).

We may assume these factors cooperate in obtaining a better result in sperm concentration improvement in patients undergoing sclerotization of their varicocele instead of the other procedures.

Though there is an evidence of correlation between patients' age and sperm parameters worsening in some studies in the literature $(26,27)$, according to other recent studies the role of age in sperm quality improvement after varicocele correction is believed to be not significant $(9,25)$. In our study the only significant trend, at linear regression estimation, is the decrease of sperm motility in relation to patients' age.

This trend is not affected by the intervention, as it remains constant after correction of varicocele, and represents the normal decrement of motility due to patient's age.

\section{Conclusions}

Varicocele treatment leads to improvement in seminal parameter examined in $73 \%$ of the cases. Both surgical and non-surgical approaches are effective, as in each group postoperative values are significantly better than preoperative ones.

Sclerotization of varicocele showed a better improvement of the postoperative sperm concentration, and is a safe and easy procedure, and should be therefore offered as a routine first line treatment to patients affected by varicocele, where the indication for treatment occurs.

Finally, even patients more than 40 years old showed a significant improvement of their sperm count and motility, and could be offered the treatment as well.

\section{REFERENCES}

1. Will MA, Swain J, Fode M, et al. The great debate: varicocele treatment and impact on fertility. Fertil Steril. 2011; 95: 841-52.

2. Belgrano E, Trombetta C, Liguori G. Scleroembolization techniques in the treatment of varicocele. Ann Urol. 1999; 33:203-9.

3. Al-Kandari AM, Shabaan H, Ibrahim HM, et al. Comparison of outcomes of different varicocelectomy techniques: open inguinal, laparoscopic, and subinguinal microscopic varicocelectomy: a randomized clinical trial. Urology. 2007; 69:417-20.

4. Shamsa A, Mohammadi L, Abolbashari M, et al. Comparison of open and laparoscopic varicocelectomies in terms of operative time, sperm parameters, and complications. Urol J. 2009; 6:170-5.

5. Dubin L, Amelar RD. Varicocele size and results of varicocelectomy in selected subfertile men with varicocele. Fertil Steril. 1970; 21:606-9.

6. Al Bakri A, Lo K, Grober E, et al. Time for improvement in semen parameters after varicocelectomy. J Urol. 2012; 187:227-31.

7. Ivanissevich $O$. Left varicocele due to reflux; experience with 4,470 operative cases in forty-two years. J Int Coll Surg. 1960; 34:742-55

8. Kocvara R, Dvorácek J, Sedlácek J, et al. Lymphatic sparing laparoscopic varicocelectomy: a microsurgical repair. J Urol. 2005; 173:1751-4

9. Liguori $G$, Ollandini $G$, Pomara $G$, et al. Role of renospermatic basal reflow and age on semen quality improvement after sclerotization of varicocele. Urology. 2010; 75:1074-8.

10. Trombetta C, Liguori G, Bucci S, et al. Percutaneous treatment of varicocele. Urol Int. 2003; 70:113-8.

11. Tauber R, Pfeiffer D. Surgical atlas varicocele: antegrade scrotal sclerotherapy. BJU Int. 2006; 98:1333-44.

12. Evers JH, Collins J, Clarke J. Surgery or embolisation for varicoceles in subfertile men. Cochrane Database Syst Rev. 2009; (1):CD000479.

13. Ficarra V, Cerruto MA, Liguori G, et al. Treatment of varicocele in subfertile men: The Cochrane Review--a contrary opinion. Eur Urol. 2006; 49:258-63.

14. Abdel-Meguid TA, Al-Sayyad A, Tayib A, Farsi HM. Does varicocele repair improve male infertility? An evidence-based perspective from a randomized, controlled trial. Eur Urol. 2011; 59:455-61.

15. Barbalias GA, Liatsikos EN, Nikiforidis G, Siablis D. Treatment of varicocele for male infertility: a comparative study evaluating currently used approaches. Eur Urol. 1998; 34:393-8.

16. Abdel-Maguid AF, Othman I. Microsurgical and nonmagnified subinguinal varicocelectomy for infertile men: a comparative study. Fertil Steril. 2010; 94:2600-3.

17. Nabi G, Asterlings S, Greene DR, Marsh RL. Percutaneous embolization of varicoceles: outcomes and correlation of semen improvement with pregnancy. Urology. 2004; 63:359-63.

18. Shlansky-Goldberg RD, VanArsdalen KN, Rutter CM, et al.Percutaneous varicocele embolization versus surgical ligation for the treatment of infertility: changes in seminal parameters and pregnancy outcomes. J Vasc Interv Radiol. 1997; 8:759-67.

19. Bechara CF, Weakley SM, Kougias P, et al. Percutaneous treatment of varicocele with microcoil embolization: comparison of treatment outcome with laparoscopic varicocelectomy. Vascular. 2009; 17(Suppl 3):S129-36.

20. Marsman JW. The aberrantly fed varicocele: frequency, veno- 
graphic appearance, and results of transcatheter embolization. AJR Am J Roentgenol. 1995; 164:649-57.

21. Gat Y, Zukerman Z, Chakraborty J, Gornish M. Varicocele, hypoxia and male infertility. Fluid Mechanics analysis of the impaired testicular venous drainage system. Hum Reprod. 2005; 20:2614-9

22. Smith $R$, Kaune H, Parodi D, et al. Increased sperm DNA damage in patients with varicocele: relationship with seminal oxidative stress. Hum Reprod. 2006; 21:986-93

23. Cuda SP, Musser JE, Belnap CM, Thibault GP. Incidence and clinical significance of arterial injury in varicocele repair. BJU Int. 2011; 107:1635-7.

24. Yamamoto M, Tsuji Y, Ohmura M, et al. Comparison of artery- ligating and artery-preserving varicocelectomy: effect on post-operative spermatogenesis. Andrologia 1995; 27:37-40.

25. Ishikawa T, Fujisawa M. Effect of age and grade on surgery for patients with varicocele. Urology 2005; 65:768-72.

26. Centola GM, Eberly S. Seasonal variations and age-related changes in human sperm count, motility, motion parameters, morphology, and white blood cell concentration. Fertil Steril. 1999; 72:803-8.

27. Sobreiro BP, Lucon AM, Pasqualotto FF, et al. Semen analysis in fertile patients undergoing vasectomy: reference values and variations according to age, length of sexual abstinence, seasonality, smoking habits and caffeine intake. Sao Paulo Med J. 2005; 123:161-6.

\section{Correspondence}

Giangiacomo Ollandini, MD (Corresponding Author) g.ollandini@gmail.com

viale Gabriele d'Annunzio 63, 34138 Trieste (Italy)

Giovanni Liguori, MD

Giorgio Mazzon, MD

Bernardino de Concilio, MD

Stefano Bucci, MD

Sara Benvenuto, MD

Emanuele Belgrano, MD

Carlo Trombetta, MD

Urologic Unit, Cattinara Hospital, University of Trieste

Via di Fiume 447 - 34149 Trieste, Italy

Stanislav Ziaran, MD

Tomáš Málek, MD

Urology Clinic, University Hospital in Bratislava,

Comenius University, Bratislava, Slovakia 